\title{
Capillary flow experiments (Capflex) for thermodynamic and kinetic characterization of protein LLPS at high throughput
}

\author{
Alexander K. Buell ${ }^{*[a]}$ \\ [a] E.G.P. Stender, Ph.D., R. K Norrild, J. A. Larsen, Professor A. K. Buell \\ Department of Biotechnology and Biomedicine \\ Technical University of Denmark - DTU \\ Søltofts Plads building 227 \\ 2800 Kgs. Lyngby \\ Denmark \\ *To whom correspondence should be addressed. E-mail: alebu@dtu.dk \\ [b] CSO H. Jensen \\ FIDA Biosystems Aps \\ Fruebjergvej 3 \\ 2100 Copenhagen
}

Emil G. P. Stender, ${ }^{[a]}$ Rasmus K. Norrild, ${ }^{[a]}$ Jacob Aunstrup Larsen ${ }^{[a]}$, Henrik Jensen ${ }^{[b]}$ and

\begin{abstract}
Liquid-liquid phase separation (LLPS) of proteins is a field of mounting importance to life sciences. The value of quantitative kinetic and thermodynamic characterization of LLPS is increasingly recognized. We present a new method, Capflex, that can easily be automated, allowing rapid and accurate quantification of key parameters for LLPS. Dilute phase concentration, relative droplet size distributions and the kinetics of droplet formation are quantified. Uniquely, the binding affinity between the polypeptide undergoing LLPS and LLPS-modulating compounds can also be determined. We applied Capflex to characterize the LLPS of Ddx4n1 and found that PEG3000 and $\mathrm{Ca}^{2+}$ promotes LLPS while ssDNA is detrimental. Furthermore, we characterized the membraneless organelle model system $\mathrm{RP}_{3}$ and provide the first experimentally recorded affinity of $\mathrm{RP}_{3}$ for $\mathrm{DNA}$. We believe the high information content and high throughput of Capflex makes it a valuable tool for characterizing biomolecular LLPS.
\end{abstract}

Biomolecular liquid-liquid phase separation (LLPS) is the spontaneous condensation of molecules into a highly concentrated

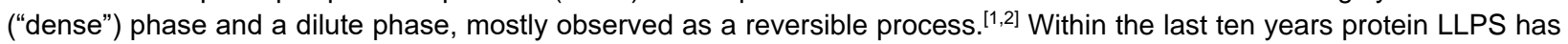
received increasing attention, due to the discovery of LLPS being a fundamental mechanism for the formation of membraneless organelles such as stress granules, and centrosomes. ${ }^{[3]}$ Thus, LLPS is a critical process to life, and is suspected to be involved in a number of neurodegenerative diseases by dysregulation of e.g. FUS, TDP-43 and a-synuclein. ${ }^{[4-}$ 6] There is therefore a great need, both in an academic as well as industrial setting, ${ }^{[7]}$ for methods that allow high throughput (HTP) characterization of biomolecular LLPS in a quantitative manner, as well as the effects of ligands and additives on lower critical concentrations (LCC). ${ }^{[8]}$ LLPS is usually characterized in vitro by fluorescence microscopy, turbidity, and absorption at $280 \mathrm{~nm} \cdot{ }^{[9]}$ Microfluidic approaches to characterize the phase diagram have also been proposed. ${ }^{[10,11]}$ However, easily approachable HTP methods for characterizing LLPS are still lacking. Here we present a fully automated method, Capflex, which is based on a commercially available instrument for flow-induced dispersion analysis (FIDA). Capflex allows straightforward characterization, in a fully thermally controlled environment, of light phase concentrations, droplet size distributions, droplet formation and maturation kinetics, as well as the affinity of the polypeptide undergoing LLPS for interaction partners that can increase or decrease the driving force for LLPS.

The protein system we have studied in the development of Capflex is the $\mathrm{n} 1$ domain of human DEAD-box helicase-4 (Ddx4), a protein involved in the formation of nuage in sperm and egg cells ${ }^{[12]}$ and suspected of being involved in ovarian cancer. ${ }^{[13]}$ The $\mathrm{n} 1$ domain of $\mathrm{Ddx} 4$ (Ddx4n1) has been shown to be responsible for the fully reversible LLPS, is intrinsically disordered and does not fold upon undergoing LLPS. ${ }^{12,14,15]}$

Capflex uses the FIDA 1 instrument, which consists of a temperature-controlled tray in a sample-handling robot holding 96well plates that are maintained above the cloud point. The sample is then loaded into a fused silica capillary kept in a temperature controlled chamber below the cloud point. Here the sample undergoes LLPS and after a time delay that can be varied with the flow rate arrives at the detector where signal spikes are recorded when droplets pass, and the baseline intensity level allows determination of the light phase concentration (Figure 1A,C,D) by standard curve calibration (Figure 1B). The method measures fluorescence compatible with YFP or Alexa488 dyes, but can also be adapted to intrinsic protein fluorescence. The detector is sufficiently sensitive so only $\mathrm{nM}$ of labelled protein is needed in the solution, which for most purposes corresponds to less than $1 \%$ of the total protein in the sample.

To test the method we first induced LLPS of Ddx4n1 by adding PEG3000 (Figure 2A). PEG is a well-known molecular crowder and has been shown to induce LLPS in other systems such as $\alpha$-synuclein. ${ }^{[4]}$ The light phase concentration decreases as a function of PEG3000 concentration (Figure 2A). As the baseline is invariant over time (Figure 1D) and the capillary chamber 
is temperature controlled the light phase concentration corresponds to the LCC for LLPS. PEG3000 has a strong effect on the LCC of Ddx4n1 as even at $2 \%$ PEG3000 the LCC is less than half of that of Ddx4n1 without additives (Figure 2A). The baseline decrease is solely due to increasing LLPS, as PEG3000 does not influence YFP fluorescence (Figure S3). Samples under identical conditions but with increased protein concentration were then measured and a LCC of $100 \mu \mathrm{M}$ was observed for all protein concentrations (Figure 2A). This result is in line with the LCC being independent of the total protein concentration at a given set of solution conditions. The absolute concentrations of light phase and dense phase do not change with total protein concentration, only their relative volume fractions. ${ }^{[9]}$ We then investigated the effect of more biologically relevant additives.

A
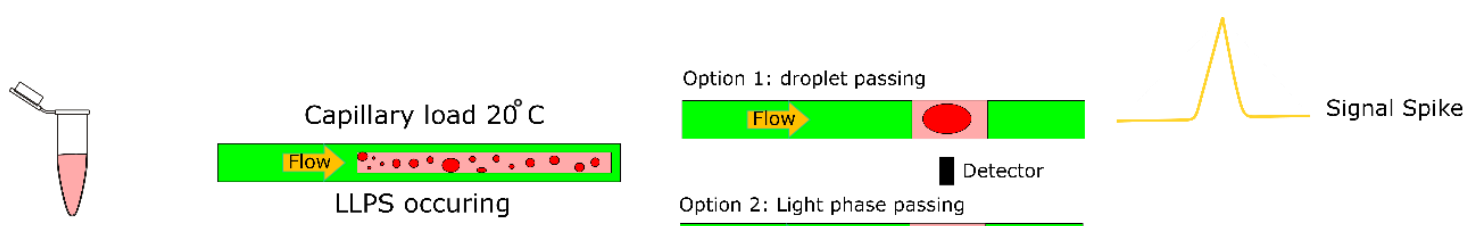

Samples in thermostatted tray above cloud point

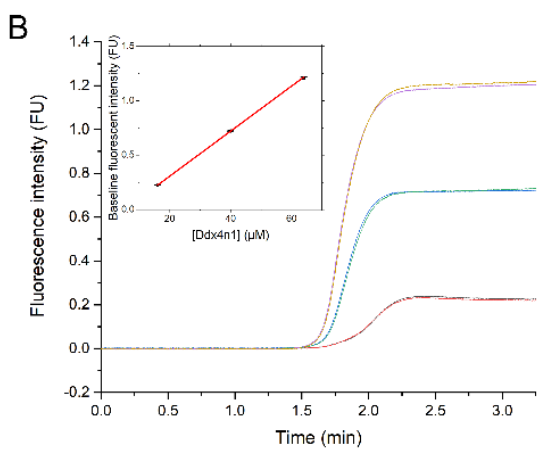

Standard curve calibration

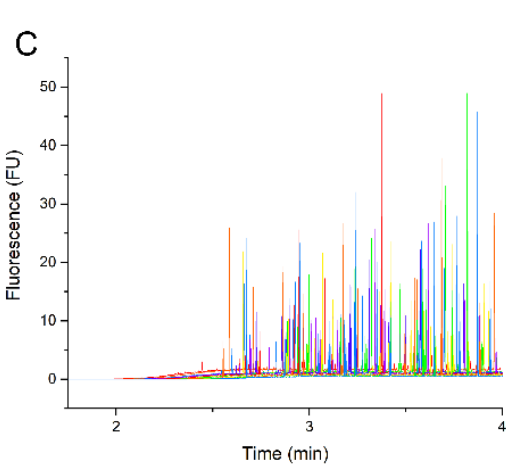

Droplet signal spikes

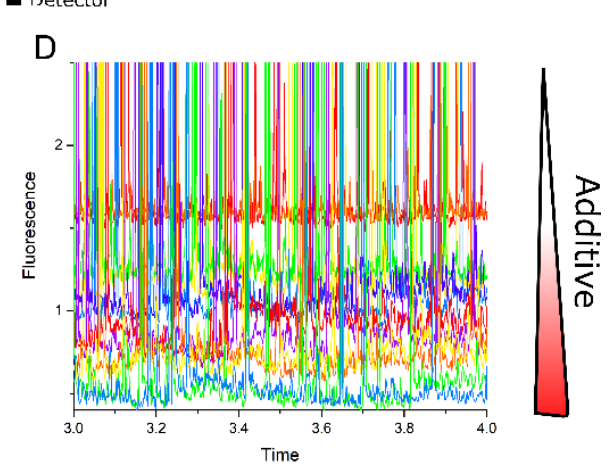

Light phase baseline signal

Figure 1. Experimental approach and example of raw data. A) Schematic representation of the experimental approach. The samples are incubated in a thermostatted tray (above the cloud point) and are then injected into the thermostatted capillary (below the cloud point) where LLPS occurs. After flowing through the $1 \mathrm{~m}$ long capillary the injected sample reaches a detector were a passing droplet causes a spike in the signal and the baseline between spikes corresponds to the light phase concentration. B) Example of standard curve calibration of the baseline. C) Example of signal spikes when droplets pass the detector. D) Baseline change in response to LLPS inducing additive.

$\mathrm{Ca}^{2+}$ is an important regulator of intracellular processes in Eukaryotes. ${ }^{[16]}$ As the concentration of $\mathrm{Ca}^{2+}$ increases, the LCC decreases in an asymptotic manner towards $65 \mu \mathrm{M}$ (Figure 2A). In agreement with similar observations by Crabtree et. al. and indicates a potential role of intracellular $\mathrm{Ca}^{2+}$ in regulation of LLPS. ${ }^{[17]}$ This effect is unlikely to be due to an increase in ionic strength as increasing $\mathrm{NaCl}$ concentration increases the lower critical concentration of $\mathrm{Ddx} 4 \mathrm{n} 1$. $^{[12]}$ In these experiments, we noted that the median peak height increases and the distribution widens as a function of total protein concentration (Figure 2B). As only the volume fraction between the two phases should change in this experiment, ${ }^{[9]}$ we hypothesized that the peak intensity distribution could be proportional to the overall droplet size distribution. However, since the Capflex experiments are performed in the laminar flow regime there could be a bias in the distribution of peak intensities, as the droplets spend different time periods in the detection volume, depending on whether they are subject to the rapid flow in the center of the capillary or slower flow at the edges (see SI for a detailed discussion). In order to quantify the magnitude of this potential bias, monodisperse polystyrene spheres with hydrodynamic diameter of 1 or $6 \mu \mathrm{m}$ were analyzed (Figure $2 \mathrm{~F}, \mathrm{G}$ ). The $1 \mu \mathrm{m}$ spheres yield an intensity distribution that is very narrow and similar to the profile observed by measuring the fluorescence intensity distribution of the spheres by microscopy (Figure S4). For the $6 \mu \mathrm{m}$ spheres the distribution is broader and the spheres saturate the detector at $50 \mathrm{FU}$, causing the distribution to be skewed. As only a few of the experimentally recorded peak intensities of Ddx4n1 approach 50 FU (Figure 2 and 3) the relative peak intensity distribution therefore closely reflects the droplet size distribution. We find that the number of detected droplets, as well as the median and width of the distribution increase as conditions become more favorable for LLPS (Figure 2 B,C,D).

We next investigated the effect of DNA on LLPS. Ddx4n1 is well known to partition single stranded DNA (ssDNA). ${ }^{[12,15]}$ We used an identical ssDNA to the one used by Nott et al. and titrated a solution of $128 \mu \mathrm{M}$ Ddx4n1 with this ssDNA (Figure 3A). 
The light phase concentration approaches the total protein concentration asymptotically as a function of ssDNA concentration, indicating that ssDNA decreases the driving force of $\mathrm{Ddx} 4 \mathrm{n} 1$ to undergo LLPS. This conclusion is further supported by the decrease of the median and width of the droplet size distributions as a function of ssDNA concentration (Figure 3B). In order to confirm that the ssDNA enters the Ddx4n1 droplets we inverted the labelling scheme and utilized a spike of Alexa488 labelled ssDNA (IDNA) in combination with unlabeled protein. Samples of $112 \mu \mathrm{M}$ Ddx4n1 was titrated with increasing concentrations of DNA and we observed that the light phase DNA concentration as a percentage of total DNA decreases asymptotically (Figure 3C). At $1 \mu \mathrm{M}$ total DNA only minute quantities of DNA have entered the droplet as most is found in the light phase. However, at 2-4 $\mu \mathrm{M}$ total DNA about $30 \%$ has entered the droplet and at $6 \mu \mathrm{M}$, where droplets are starting to dissolve (for $112 \mu \mathrm{M} d d x 4 \mathrm{n} 1$, Figure $3 \mathrm{C}$ ) about $60 \%$ of the DNA is found in the light phase. The fact that only few droplets are observed in this condition (Figure 3D) indicates that the observed droplets are very enriched in DNA and that in addition many droplets are too small to be clearly resolved as such by the FIDA 1 instrument. The latter is likely since $112 \mu \mathrm{M}$ Ddx4n1 is close to the critical concentration for LLPS at $6 \mu \mathrm{M}$ DNA (Figure 3C, Figure S5). The droplets are entirely dissolved at high DNA concentration of $26.6 \mu \mathrm{M}$ (Figure $3 \mathrm{C}$ ), where all the DNA is again found to be in the dilute phase. In order to understand how such a highly sub-stoichiometric concentration of DNA is able to dissolve the droplets, we determined the relative enrichment factor of Ddx4n1 to DNA in the droplets. To this end, we analyzed samples with identical concentrations (112 $\mu \mathrm{M}$ $\mathrm{Ddx} 4 \mathrm{n} 1$ and 3.3 $\mu \mathrm{M}$ ssDNA) with the only difference being whether there is a spike of IDNA or Ddx4n1-YFP (Figure 3F, Figure S6). In this case the volume fraction of dense phase, as well as the concentrations of DNA and protein in the dense phase can be expected to be the same.

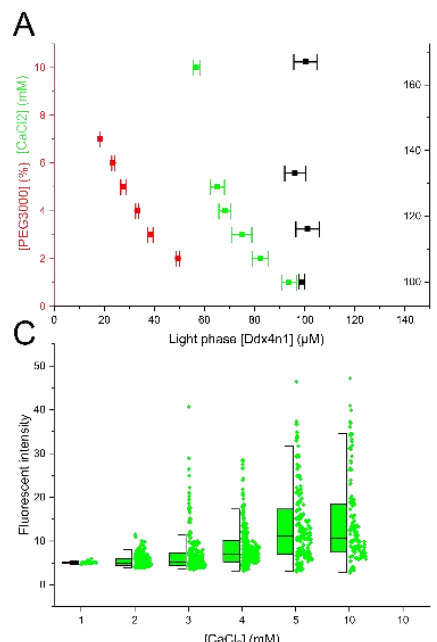

B
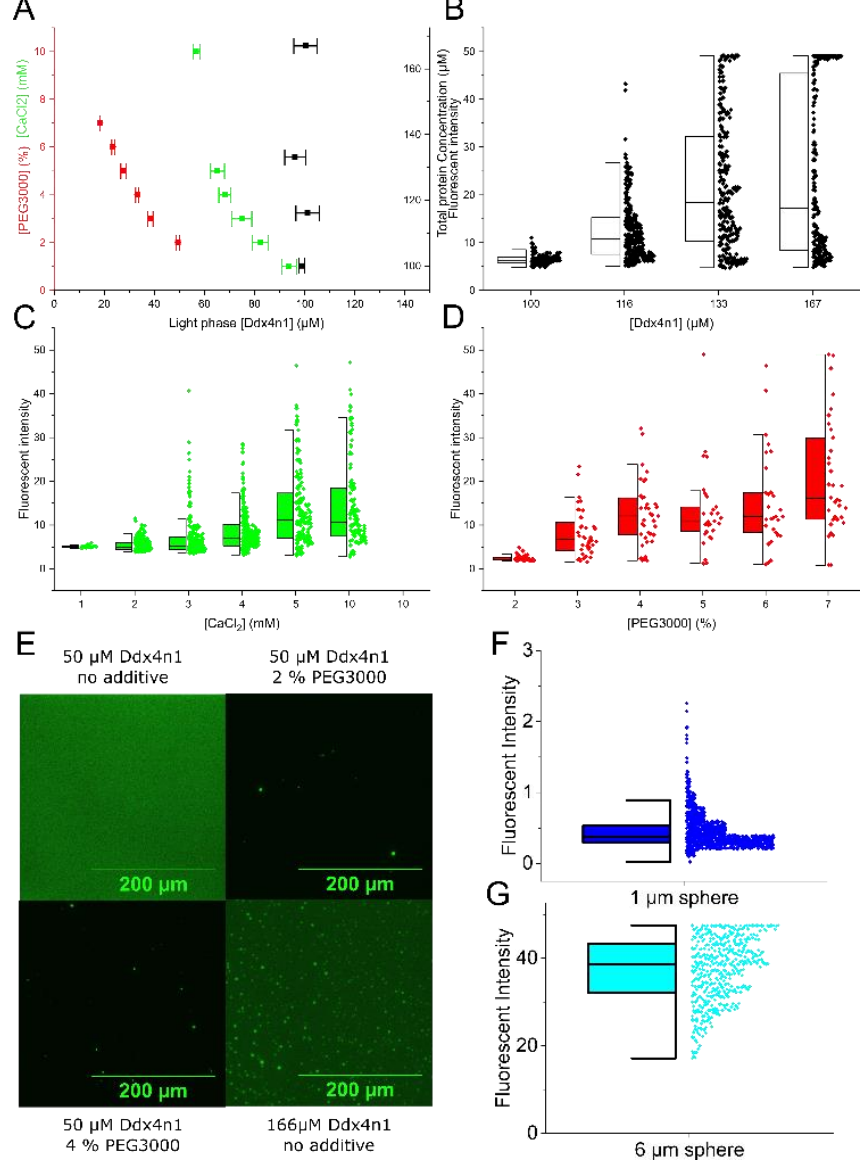

Figure 2. Lower critical concentration determination and droplet size distributions. A) Light phase concentration as a function of additives at $20^{\circ} \mathrm{C}$ (green) $\mathrm{Ca}^{2+}$ in mM with $100 \mu \mathrm{M}$ Ddx4n1, (red) PEG3000 in \% with $50 \mu \mathrm{M}$ Ddx4n1 and (black) Ddx4n1 in $\mu \mathrm{M}$. B) Peak intensity distribution as a function of $\mathrm{Ddx} 4 \mathrm{n} 1$ concentration, C) as a function of $\mathrm{Ca}^{2+}, \mathrm{D}$ ) as a function of PEG3000. E) Fluorescence microscope images of Ddx4n1 with and without PEG3000 at a 40x magnification. The pictures are recorded at different exposures to ensure that the maximum amount of droplets are visible. F) Peak intensity distribution of $1 \mu \mathrm{m}$ fluorescent polystyrene spheres. G) Peak intensity distribution of $6 \mu \mathrm{m}$ fluorescent polystyrene spheres. 
We estimated the relative enrichment by calculating the ratio between the integral of the signal spikes and the baseline integral for both label conditions and then calculated the ratio between these two ratios. This procedure eliminates the need to know the volume fraction of dense phase, as it cancels out of the ratio of ratios. This procedure assumes that neither the fluorescence of the YFP nor of the Alexa tag is significantly different outside and inside the droplets. ${ }^{[15]}$ We obtain an enrichment factor between protein to DNA in the droplets of $1.5 \pm 0.26$. DNA and protein are therefore present in the dense phase at a similar stoichiometry to the overall stoichiometry, in this case a ca. 35 fold excess of protein. In order to understand better how a strongly substoichiometric concentration of DNA is able to significantly destabilize the droplet phase of Ddx4n1 (Figure 3A,B,D), we measured the affinity of Ddx4n1YFP for ssDNA. Interactions between the positively charged arginine and the negative charge on ssDNA could potentially screen the Phe-Arg interactions important for Ddx4n1 LLPS. ${ }^{14]}$ We took advantage of the inbuilt function of the FIDA 1 instrument to measure the affinity and complex size of an indicator and analyte molecule. ${ }^{[18]}$ The ability to obtain interaction affinity data is a unique added benefit of the FIDA 1-based Capflex method. We chose to utilize Ddx4n1-YFP as indicator as the concentration can be kept in the nM range preventing LLPS and the ssDNA as analyte. The interaction between Ddx4n1-YFP is weak with a $\mathrm{K}_{d}$ of $50.9 \pm 11.1 \mu \mathrm{M}$ (Figure 3E). The observed increase in size, from ca. $3.5 \mathrm{~nm}$ to 7 or more $\mathrm{nm}$ suggests that more than a single DNA molecule interacts with one molecule of Ddx4n1, which is why a 1:2 binding model was applied (see SI for details). Interestingly, the dissociation constant between DNA and $\mathrm{Ddx} 4 \mathrm{n} 1$ far exceeds the concentrations where destabilizing effects for LLPS are observed. This result can be rationalized if the collective nature of the phenomenon of LLPS is considered, in which multivalency is crucial. Within a condensate droplet, each molecule of DNA is able to simultaneously interact with several molecules of Ddx4n1, and is therefore able to disrupt a multitude of interactions necessary for LLPS.

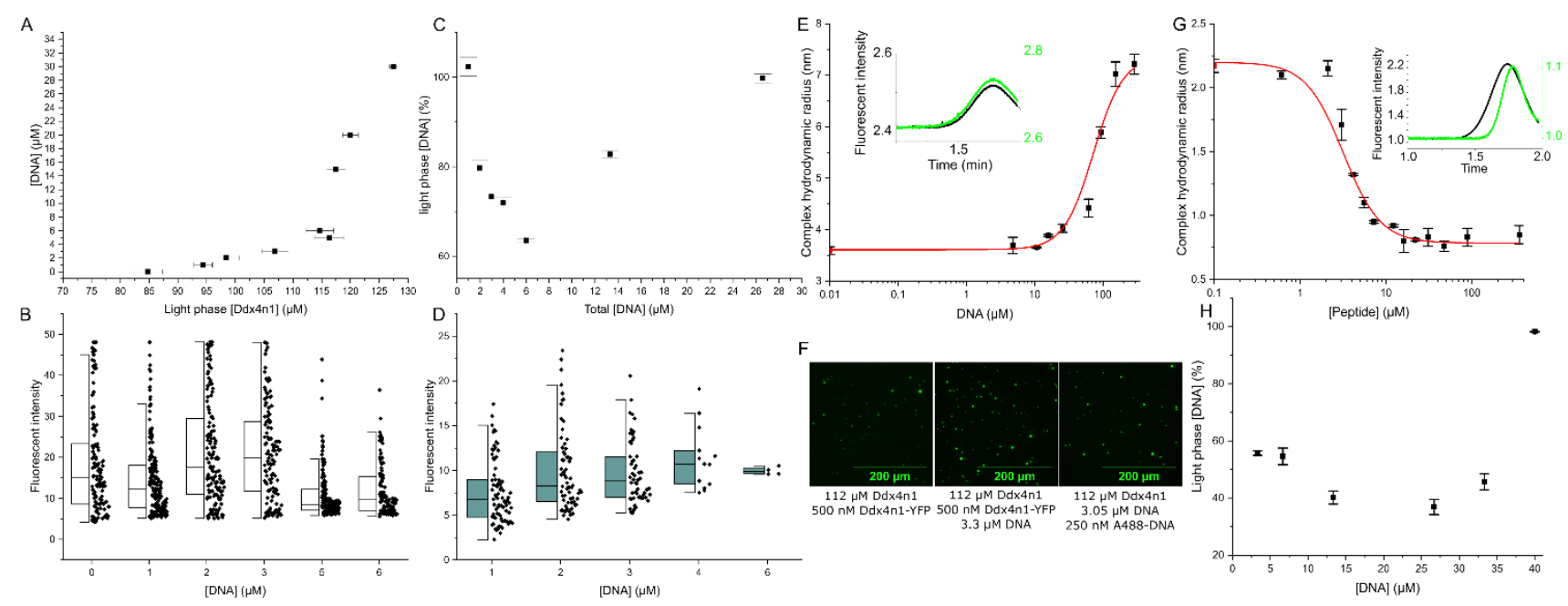

Figure 3. FIDA analysis of the effect of ssDNA on LLPS. A) Critical concentration of a $128 \mu \mathrm{M}$ Ddx4n1 as a function of DNA added. B) Droplet size distribution of $128 \mu \mathrm{M}$ Ddx4n1 as a function of DNA added. C) Light phase DNA concentration as a function of total DNA added to $112 \mu \mathrm{M}$ Ddx4n1. D) Droplet size distribution of $112 \mu \mathrm{M} \mathrm{Ddx} 4 \mathrm{n} 1$ as a function total DNA added with $0.5 \%$ spike of IDNA. E) Affinity determination between Ddx4n1-YFP and ssDNA. The insert is 0 (black) and $63 \mu \mathrm{M}$ (green) DNA added. The red point is $0 \mu \mathrm{M}$ DNA added. F) Microscopy images of $112 \mu \mathrm{M}$ Ddx4n1 with either Ddx4n1-YFP or A488-DNA added as the fluorescent tracer. G) Affinity determination of $\mathrm{RP}_{3}$ peptide and ssDNA the red point is $0 \mu \mathrm{M}$ DNA the insert is the Taylor grams with 0 (black) and $358 \mu \mathrm{M}$ (green) DNA added. $\mathrm{H}$ ) Critical concentration of ssDNA in coacervation with $\mathrm{RP}_{3}$ peptide as a function of ssDNA added.

We also applied our approach to a small peptide system undergoing LLPS through coacervation to further demonstrate its versatility. The synthetic $\mathrm{RP}_{3}$ peptides have previously been applied as a model for intracellular membraneless organelles as they form coacervates with $\mathrm{RNA}^{[19]}$ as well as with ssDNA. ${ }^{[20]}$ As ssDNA is added the amount of ssDNA in the light phase decreases down to $37 \%$ at $26 \mu \mathrm{M}$ DNA added corresponding closely to the optimal mass fraction of $\mathrm{RP}_{3} / \mathrm{ssDNA}_{\mathrm{s}} 1$ reported previously. ${ }^{[20]}$ Much as observed with Ddx4n1 when the ssDNA concentration becomes high enough the droplets eventually dissolve entirely (Figure 3H, Figure S7, S8) in agreement with previous reports. ${ }^{[20]}$ The affinity of DNA for $\mathrm{RP}_{3}$ was measured similarly to the affinity of Ddx4n1 for DNA but this time we used the Alexa-448 labelled DNA as the indicator. The hydrodynamic radius of the IDNA is $2.3 \pm 0.2 \mathrm{~nm}$ in the unbound form, in close agreement with the expected value of $2.2 \mathrm{~nm} .^{[21]}$ As peptide is titrated in, the apparent hydrodynamic radius decreases and eventually approaches $0.8 \pm 0.0 \mathrm{~nm}$ indicative of a strong 
collapse of the DNA structure, highlighting the strong effect of charge neutralization of the $10 \mathrm{kDa}$ negatively charged DNA by the $1 \mathrm{kDa} \mathrm{RP}_{3}$ peptide. The difference in size of the IDNA and $\mathrm{RP}_{3}$ peptide makes it likely that more than one peptide molecule can bind to each DNA molecule. We therefore fitted a one to two binding model to the data (Figure $3 G$ ) yielding a $K_{D}$ of $5.3 \pm$ $0.4 \mu \mathrm{M}$. This result highlights the difference in interaction strength with DNA between the coacervation dependent $\mathrm{RP}_{3}$ peptide and Ddx4n1 (Figure 3E,G). To the best of our knowledge, the FIDA approach to characterizing LLPS is the only method that directly yields the affinity between interaction partners undergoing LLPS alongside relative droplet size distributions, light phase concentration as well as the complex size.

We then investigated whether our methodology could be used to monitor initial droplet formation kinetics, something that is currently difficult to measure at HTP. We used $50 \mu \mathrm{M}$ Ddx4n1 with increasing amounts of PEG3000 to demonstrate the ability of Capflex to resolve the kinetics of droplet formation with the samples initially kept at $55^{\circ} \mathrm{C}$ above the cloud point and the capillary at $20^{\circ} \mathrm{C}$. The capillary was loaded with sample at the highest possible flow rate and thus filled within 15 sec, followed by a slow injection during which peak intensity development was followed over time (Figure 4). At the edge of the binodal line with $2 \% \mathrm{PEG}$ and $50 \mu \mathrm{M} \mathrm{Ddx} 4 \mathrm{n} 1$ there is a significant delay of $30 \mathrm{~s}$ before peaks are detected and the signal reaches a steady state after about $1.2 \mathrm{~min}$ at average peak intensity of 1.2 (Figure 4A,D). As the PEG3000 concentration increases and the reaction conditions correspond to a larger driving force for LLPS, the increase in average peak intensity is much more rapid (Figure 4B,C,D) with maximum peak intensities being reached after 1.5 min, indicating that the system approaches spinodal decomposition rather than featuring nucleated behavior. ${ }^{[9]}$
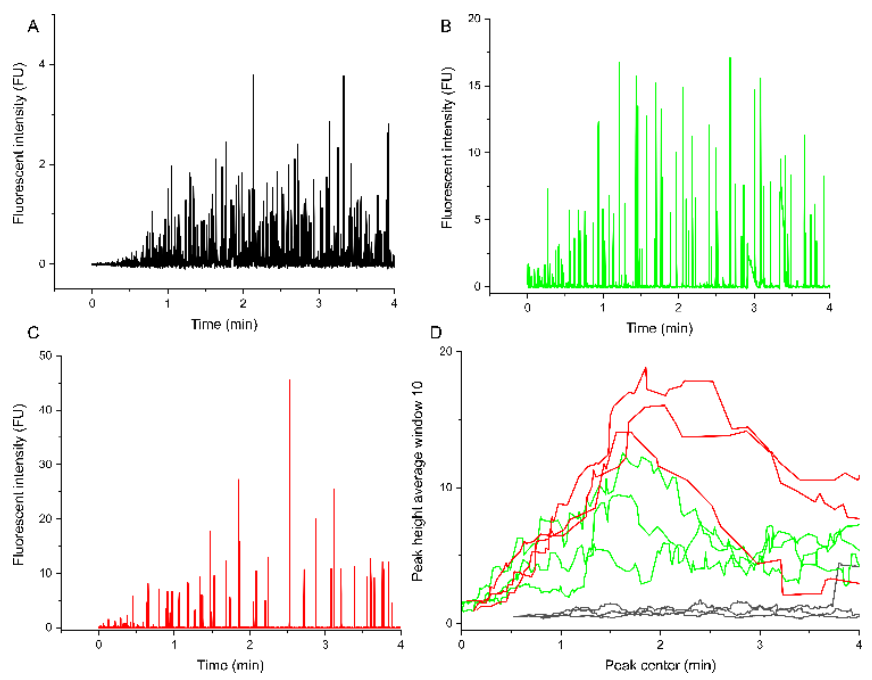

Figure 4. Droplet formation kinetics of $50 \mu \mathrm{M}$ Ddx4n1 as a function of PEG3000 concentration. A) Peak intensity development of $50 \mu \mathrm{M}$ Ddx4n1 and $2 \%$ PEG3000. B) $3 \%$ PEG3000. C) $4 \%$ PEG3000. D) Triplicate of peak intensity development with an averaging window of 10 peaks $2 \%$ PEG3000 (black), $3 \%$ PEG3000 (green), $4 \%$ PEG3000 (red).

In conclusion, we have developed a fully automated HTP method for characterizing LLPS, Capflex, that provides light phase concentrations, relative droplet size distributions, relative droplet formation kinetics, affinity between interaction partners undergoing LLPS as well as their experimental complex size, all with a single experimental setup. We applied the method to both a biologically relevant protein and a peptide model system. We believe that this fully automated and off-the-shelf method for characterizing biomolecular LLPS will be of great value to the research community interested in LLPS.

\section{Acknowledgements}

Charlotte O'Shea from University of Copenhagen is thanked for donating the TEV-MBP plasmid. We thank Dr. Tim Nott (Department of Biochemistry) and Dr. Andrew Baldwin (Department of Chemistry), both at Oxford University, for donating the PET-30M plasmids containing the GST-Ddx4n1 fusion constructs and for help with protein production. Kristina Mielec is thanked for technical assistance. EGPS, RN, JL and AKB would like to acknowledge the Novo Nordisk Foundation for funding (Grant number: NNFSA170028392). The Carlsberg Foundation is acknowledged for funding the FIDA 1 instrument with a 
grant to Andreas H. Lausten (Grant number: CF19-0055). Funding from Novo Nordisk Foundation (grant NNFOC0055625) for the infrastructure "Imaging microbial language in biocontrol (IMLiB)" is acknowledged.

\section{Conflict of interest}

Henrik Jensen is the CSO of FIDA Biosystems. The authors declare no conflict of interest.

Keywords: Liquid-liquid phase separation • Protein-protein Interactions • Partitioning $•$ Coacervation $•$ High Through-put Screening.

[1] Zaslavsky, Ferreira, Uversky, Biomolecules 2019, 9, 473.

[2] S. S. Ribeiro, N. Samanta, S. Ebbinghaus, J. C. Marcos, Nat. Rev. Chem. 2019.

[3] V. N. Uversky, Curr. Opin. Struct. Biol. 2017, 44, 18-30.

[4] S. Ray, N. Singh, R. Kumar, K. Patel, S. Pandey, D. Datta, J. Mahato, R. Panigrahi, A. Navalkar, S. Mehra, L. Gadhe, D. Chatterjee, A. S. Sawner, S. Maiti, S. Bhatia, J. A. Gerez, A. Chowdhury, A. Kumar, R. Padinhateeri, R. Riek, G. Krishnamoorthy, S. K. Maji, Nat. Chem. 2020, 12, 705-716.

[5] E. S. Arnold, S. C. Ling, S. C. Huelga, C. Lagier-Tourenne, M. Polymenidou, D. Ditsworth, H. B. Kordasiewicz, M. McAlonis-Downes, O. Platoshyn, P. A. Parone, S. Da Cruz, K. M. Clutario, D. Swing, L. Tessarollo, M. Marsala, C. E. Shaw, G. W. Yeo, D. W. Cleveland, Proc. Natl. Acad. Sci. U. S. A. 2013, 110.

[6] H. Deng, K. Gao, J. Jankovic, Nat. Rev. Neurol. 2014, 10, 337.

[7] E. Dolgin, Nat. Biotechnol. 2021, 39, 123-125.

[8] K. M. Ruff, F. Dar, R. V Pappu, Proc. Natl. Acad. Sci. 2021, 118.

[9] S. Alberti, A. Gladfelter, T. Mittag, Cell 2019, 176, 419-434.

[10] W. E. Arter, R. Qi, G. E. Krainer, T. J. Welsh, Y. Xu, P. S. George-Hyslop, S. Alberti, T. Knowles, bioRxiv 2020, 2020.06.04.132308.

[11] M. Linsenmeier, M. R. G. Kopp, S. Stavrakis, A. de Mello, P. Arosio, Biochim. Biophys. Acta - Mol. Cell Res. $2021,1868$.

[12] T. J. Nott, E. Petsalaki, P. Farber, D. Jervis, E. Fussner, A. Plochowietz, T. D. Craggs, D. P. Bazett-Jones, T. Pawson, J. D. Forman-Kay, A. J. Baldwin, Mol. Cell 2015, 57, 936-947.

[13] Y. Chen, Z. Sun, J. Xu, P. Wang, J. Tang, X. Shi, J. Liu, F. Ren, L. Xu, Oncol. Lett. 2018, 16, 905-909.

[14] J. P. Brady, P. J. Farber, A. Sekhar, Y. H. Lin, R. Huang, A. Bah, T. J. Nott, H. S. Chan, A. J. Baldwin, J. D. Forman-Kay, L. E. Kay, Proc. Natl. Acad. Sci. U. S. A. 2017, 114, E8194-E8203. 
[15] T. J. Nott, T. D. Craggs, A. J. Baldwin, Nat. Chem. 2016, 8, 569-575.

[16] E. A. Permyakov, R. H. Kretsinger, J. Inorg. Biochem. 2009, 103, 77-86.

[17] M. D. Crabtree, J. Holland, P. Kompella, L. Babl, N. Turner, A. J. Baldwin, T. J. Nott, bioRxiv 2020.

[18] H. Jensen, J. Østergaard, J. Am. Chem. Soc. 2010, 132, 4070-4071.

[19] W. M. Aumiller, C. D. Keating, Nat. Chem. 2016, 8, 129-137.

[20] P. R. Banerjee, A. N. Milin, M. M. Moosa, P. L. Onuchic, A. A. Deniz, Angew. Chemie - Int. Ed. 2017, 56, 11354-11359.

[21] A. I. Díez, J. M. Ortiz-Guerrero, A. Ortega, M. Elías-Arnanz, S. Padmanabhan, J. García De La Torre, Eur. Biophys. J. 2013, 42, 463-476. 